Desigualdad de

\title{
oportunidades y
}

movilidad

intergeneracional

en Canarias

Resumen ejecutivo

Gustavo Alberto Marrero Díaz (Coord.)

Moisés Betancort Montesinos

Leopoldo José Cabrera Rodríguez

Sara Darias Curvo

Carmen Nieves Pérez Sánchez

Juan Gabriel Rodríguez Hernández

Daniel Sánchez Rodríguez 


\title{
Desigualdad de oportunidades y movilidad intergeneracional en Canarias
}

\section{Resumen ejecutivo}

\author{
Gustavo Alberto Marrero Díaz (Coord.) \\ Moisés Betancort Montesinos \\ Leopoldo José Cabrera Rodríguez \\ Sara Darias Curvo \\ Carmen Nieves Pérez Sánchez \\ Juan Gabriel Rodríguez Hernández \\ Daniel Sánchez Rodríguez
}

San Cristóbal de La Laguna, noviembre de 2017

DOI: $\underline{10.25145 / \text { r.cedesog.2018.002 }}$

\section{CajaCanarias}

El presente documento constituye un resumen ejecutivo del Informe Desigualdad de oportunidades y movilidad intergeneracional en Canarias, elaborado por el equipo de investigadores del CEDESOG encabezado por Gustavo Alberto Marrero Díaz, en el marco del proyecto de investigación "Desigualdad de Oportunidades en Canarias: Incidencia de la inclusión laboral, la educación y la salud" (referencia CSOCTRA07), financiado por la Fundación Cajacanarias, dentro de su Programa de Proyectos de Investigación en I+D y Ciencias Sociales. Sin el apoyo financiero, la elaboración de este Informe no hubiera sido posible, por lo que todo el equipo del proyecto agradece a la Fundación Cajacanarias su apoyo y el compromiso social en favor de grupos de investigación de las universidades canarias para la ejecución de proyectos competitivos con impacto en la región. 
El Centro de Estudios de Desigualdad Social y Gobernanza de la Universidad de La Laguna (CEDESOG) es un centro interuniversitario y multidisciplinar creado en julio de 2016 (mediante acuerdo 3/CG 14-7-2016) dedicado a la investigación, análisis, coordinación, realización y divulgación científica de estudios académicos sobre desigualdad social, desigualdad de oportunidades y movilidad social intergeneracional, en los ámbitos -principalmente- de la Economía, la Sociología, la Educación o la Salud Pública. El centro, de este modo, reúne a expertos en desigualdad de prestigio procedentes de las dos universidades canarias (ULL y ULPGC), además de otras universidades y centros nacionales (la Universidad Complutense de Madrid o la Universidad de Oviedo, entre ellos) e internacionales (London School of Economics), además de profesionales del sector de la salud pública. El potencial del centro alcanza desde el asesoramiento y la evaluación de políticas públicas para la lucha contra la pobreza y la desigualdad (en colaboración con agentes como las Administraciones Públicas, ONGs o agencias de desarrollo local, etc.) hasta la creación de producción científica, de investigación o divulgación relacionada con la pobreza, la desigualdad o la gobernanza, contribuyendo a dar visibilidad a dichos problemas no sólo en el ámbito canario, sino en otras regiones de características similares, como la Macaronesia.

Director: Gustavo Alberto Marrero Díaz

Sub-directora: Sara Darias Curvo

Secretario: Leopoldo José Cabrera Rodríguez

Centro de Estudios de Desigualdad Social y Gobernanza de la ULL (CEDESOG)

Secretaría de la Facultad de Economía, Empresa y Turismo

Camino de la Hornera S/N

38071, San Cristóbal de La Laguna

Teléfono: 922317123

E-mail: cedesog@ull.edu.es

En las redes sociales: @CedesogTF (acebook y Twitter) 


\title{
Desigualdad de oportunidades y movilidad intergeneracional en Canarias
}

\section{Resumen ejecutivo}

\author{
San Cristóbal de La Laguna, noviembre de 2017
}

\begin{abstract}
Resumen:
A pesar de la mejoría en los indicadores macroeconómicos que Canarias experimenta desde 2014, los datos muestran que la recuperación aún no ha llegado a una gran parte de la población. Con importantes índices de concentración en la renta, además de un preocupante $44,6 \%$ de la población en riesgo de la pobreza o exclusión social (indicador AROPE), Canarias está entre las regiones españolas (y europeas) más desiguales. Luchar contra la desigualdad de oportunidades, originada por las diferencias en las circunstancias, es uno de los mayores retos que los países deben afrontar en los próximos años. Reducir la desigualdad de oportunidades es necesario para construir sociedades más justas y economías más eficientes, en donde los ciudadanos/as tengan incentivos a participar y aprovechar plenamente sus conocimientos y capacidades. Reducir la inmovilidad intergeneracional (la dependencia entre los logros del individuo y los de sus padres) es clave para combatir la desigualdad de oportunidades. El informe Desigualdad de oportunidades y movilidad intergeneracional en Canarias, realizado por el Centro de Estudios de Desigualdad Social y Gobernanza de la Universidad de La Laguna (CEDESOG) analiza la influencia, las interrelaciones y los canales de transmisión tanto de la desigualdad de oportunidades como de la inmovilidad intergeneracional en las islas, teniendo en cuenta variables como la educación, el estado de salud, la ocupación o la renta.
\end{abstract}

Palabras clave: Desigualdad, desigualdad de oportunidades, inmovilidad intergeneracional, Canarias

\begin{abstract}
:
Despite the improvement in the macroeconomic indicators that the Canary Islands have experienced since 2014 , the data show that recovery has not reached yet a large part of the population. With significant rates of income concentration, as well as a worrying $44.6 \%$ of the population at risk of poverty or social exclusion (AROPE indicator), the Canary Islands are among the most unequal Spanish (and European) regions. Fighting against inequality of opportunity, caused by differences on the circumstances, is one of the most challenging issues that countries will have to face in the next years. Reducing inequality of opportunity is necessary to build fairer societies and more efficient economies, where individuals have incentives to participate and to reduce employ all its knowledge and capabilities. Lowering intergenerational immobility (the degree of dependence between individuals' attainments and its parent ones) is a key issue to reduce inequality of opportunity. The report Inequality of opportunity and intergenerational mobility in the Canary Islands, carried out by University of La Laguna's Research Centre of Social Inequality and Governance (CEDESOG) analyzes the influence, the interdependence and the transmission channels of both inequality of opportunity and intergenerational mobility in the islands, taking into account variables such as education, heath, occupation and income.
\end{abstract}

Key-words: Inequality, Inequality of opportunity, intergenerational mobility, Canary Islands. 


\section{Índice}

1. Introducción: Disparidad, desigualdad y fractura social ...........................................................

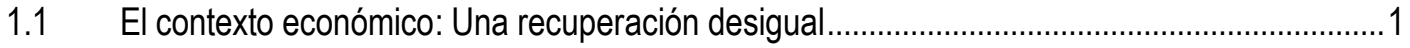

1.2 Desigualdad de oportunidades y prosperidad económica …......................................................

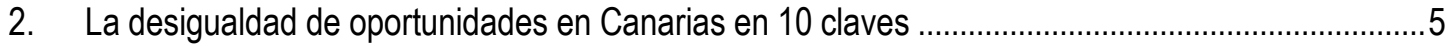

3. 10 recomendaciones de política para luchar contra la desigualdad de oportunidades en Canarias.

4. Bibliografía

\section{Índice de gráficos}

GRÁFICO 1: Evolución del PIB y el PIB per cápita (PIBpc) en Canarias y España (2008 = 100), 2006-2016

GRÁFICO 2: Evolución de la población ocupada y desempleada en Canarias y España (2008 = 100, eje primario) y tasa de paro en Canarias y España (eje secundario), 2006-2016.

GRÁFICO 3: Evolución de la renta por hogar en Canarias y España (2008 $=100$, eje primario) y proporción de la renta por hogar en Canarias respecto a España, y tasa de crecimiento de la renta por hogar en Canarias y España (\%, eje secundario)

GRÁFICO 4: Distribución de la renta neta total de los individuos, según el decil de renta al que pertenecen

GRÁFICO 5: Probabilidad condicionada de que el individuo alcance un determinado nivel de estudios, condicionada al nivel educativo de los padres (ambos) 


\section{Introducción: Disparidad, desigualdad y fractura social}

\subsection{El contexto económico: Una recuperación desigual}

Canarias inicia en 2017 el que según las previsiones de diversos organismos es el cuarto año de recuperación económica. Los principales indicadores macroeconómicos, como el PIB o la tasa de desempleo, comienzan a recuperarse a partir del año 2014, tras dejar atrás el impacto de la crisis subprime originada en EE.UU. y la segunda recesión ocasionada por la crisis de deuda soberana europea. En términos de producción, este hecho se constata con la vuelta en 2016 a los niveles de producción del año 2008 (Gráfico 1), o en el comienzo del decline en la tasa de paro (Gráfico 2). Las previsiones, de acuerdo con organismos como Ceprede (Centro de Predicción Económica), BBVA Research (2017) o CEOE-Tenerife, todos ellos, situando a las islas por encima de la media nacional, con una tasa de crecimiento de entre el $3,2 \%$ y $3,5 \%$.

Sin embargo, no todo son luces en esta recuperación económica en Canarias. A pesar de volver a la senda del crecimiento, este retorno se ha realizado con una economía cada vez menos diversificada y dependiente cada vez más del turismo y el sector servicios. Canarias tampoco ha recuperado los niveles de renta per cápita previos a la crisis; es más, la renta por habitante en Canarias es en la actualidad un 7\% inferior a lo que lo era en 2008 (llegando a perder hasta un 12\% entre 2013 y 2014) y ha consolidado la tendencia de divergencia hacia la renta por habitante nacional iniciada a mediados de los 90 . Hoy, la renta por habitante en Canarias es un $82 \%$ de la nacional y apenas un $66 \%$ de la comunitaria. En términos de empleo, la situación es similar, pese a que Canarias haya recuperado prácticamente, en el tercer trimestre de 2017, el número de ocupados del primer trimestre de 2008. La tasa de paro se mantiene en el entorno del $21 \%$ en el tercer trimestre de 2017 (una media en lo que llevamos de año de, aproximadamente el $24 \%$, pero que llegó a alcanzar el $34 \%$ en 2013), siendo, en todo caso, uno de los valores más elevados de Europa. La Gran Recesión supuso para muchos de los que llegaron a las islas buscando una oportunidad de progreso, el billete de retorno a sus países de origen, 0 en el caso de muchos canarios y canarias (sobre todo, los más jóvenes), abandonar las islas en busca de oportunidades en el extranjero.

GRÁFICO 1: Evolución del PIB y el PIB per cápita (PIBpc) en Canarias y España (2008 = 100), 20062016

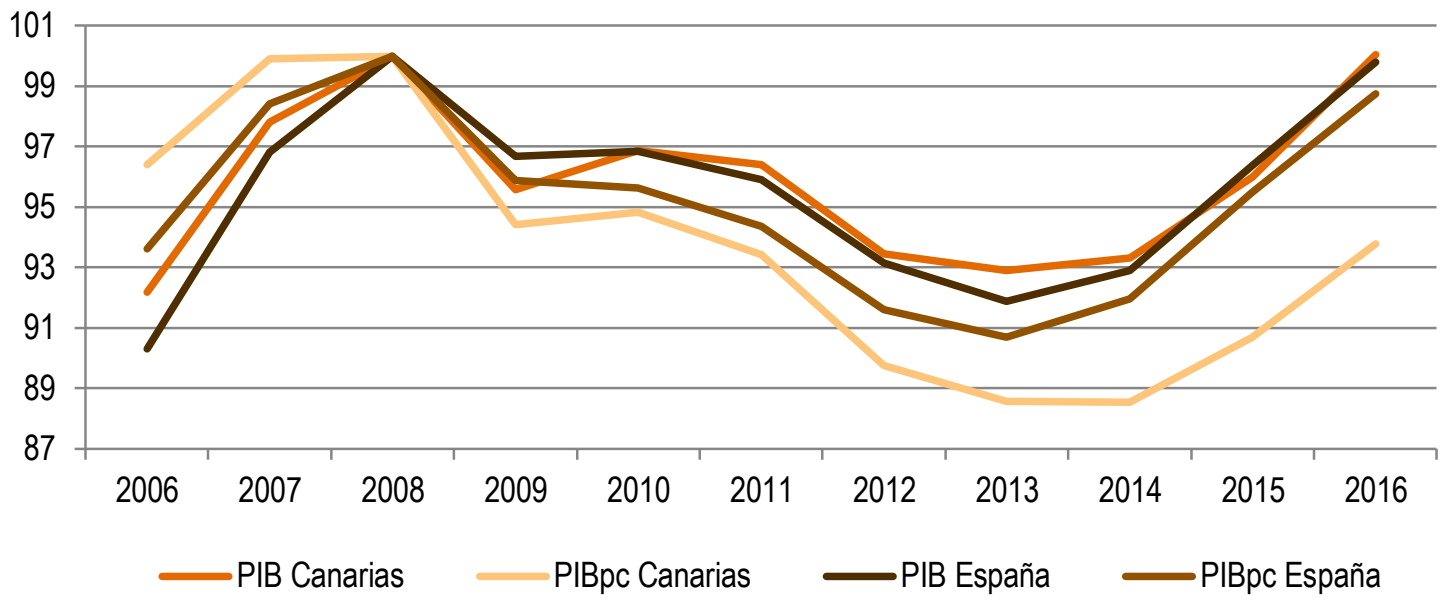

FUENTE: ISTAC. Contabilidad Regional de España / Series anuales del PIB y sus componentes por CC.AA 
GRÁFICO 2: Evolución de la población ocupada y desempleada en Canarias y España (2008 $=100$, eje primario) y tasa de paro en Canarias y España (eje secundario), 2006-2016

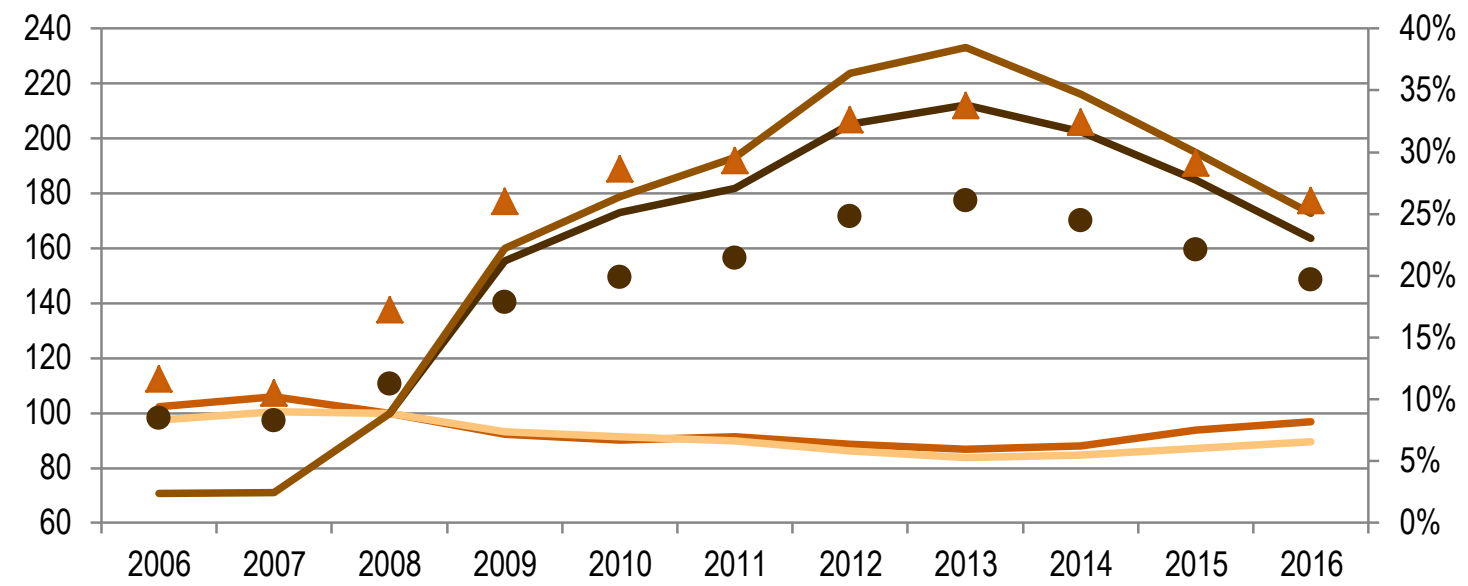

Ocupados Canarias
Parados España

Ocupados España

- Parados Canarias

FUENTE: INE. Encuesta de Población Activa. Resultados por CC.AA.

NOTA: El cálculo de los indicadores de población ocupada y desempleada se ha realizado utilizando el promedio de los datos trimestrales ofrecidos por el INE, hasta el último año completo $(20$

Cabe entonces preguntarse, una vez expuesto esto, y teniendo en cuenta que el sector turístico, buque insignia de nuestra economía, sigue rompiendo récords año tras año, si la recuperación está llegando a los hogares y si las familias residentes en las islas están comenzando a notar esta recuperación. Los datos, desafortunadamente no parecen indicarlo. La renta de las familias canarias es en 2016 un 10\% inferior a lo que lo era en 2008 (Gráfico 3), al tiempo que, tal y como comentábamos anteriormente, se ha consolidado la divergencia con respecto de la renta media de las familias españolas (los ingresos de las familias canarias son un $83 \%$ de los de una familia 'promedio' del resto del Estado). La renta disponible bruta per cápita de los hogares canarios se sitúa en el mismo entorno del $83 \%$ del promedio nacional en la última estimación (2014) del INE (2016), lo cual supone un descenso de casi $1.000 €$ desde 20101. El salario medio por trabajador en Canarias es en 2015 de 19.856,61€, el 85\% de la media nacional, más de $4.000 €$ más bajo en el caso de los trabajadores/as con contrato indefinido (el $83 \%$ de un trabajador residente en otra región del Estado). Del mismo modo, la distancia entre los percentiles extremos de renta (10 y 90; el 10\% que menos ingresa y el 10\% que más) se han ensanchado desde el 3,72 hasta casi 5 veces el ingreso mensual neto de los primeros. Esta trayectoria de divergencia, junto con la menor renta por habitante, llevan a una mayor incidencia de las tasas de pobreza relativa en el archipiélago.

Teniendo en cuenta los datos aportados en los párrafos anteriores, y el patrón de especialización canario, lo cierto es que la baja productividad de la economía, la incidencia de una de las tasas de temporalidad más altas del Estado (según Fedea, del 32,5\%, más de 6 pp. por encima de la media nacional), los bajos salarios y una tasa de paro que hasta no hace mucho, afectaba a 1 de cada 3 trabajadores/as, resultados como los recientemente revelados por el informe de la Red Europea de Lucha Contra la Pobreza y la Exclusión Social (EAPN-ES), en el cual se refleja un muy preocupante $44,6 \%$ de la población de las islas en riesgo de pobreza o exclusión social o un fenómenos como el de la pobreza laboral, que ya afecta a 1 de cada 5 trabajadores en las islas, o la disparidad y cada vez mayor

\footnotetext{
${ }^{1}$ La renta percibida por los hogares por su participación en las actividades productivas, una vez se incorpora la acción redistributiva del Estado (impuestos directos, cotizaciones y prestaciones sociales), antes de incluir las transferencias sociales en especie (educación, sanidad o protección social). Para más información, consultar Padrón Marrero, Martínez García (Dirs.) et al. (2016).
} 
concentración en el reparto de la renta, desgraciadamente no nos son extraños, teniendo en cuenta los problemas estructurales aún persistentes en la economía canaria².

GRÁFICO 3: Evolución de la renta por hogar en Canarias y España $(2008=100$, eje primario $)$ y proporción de la renta por hogar en Canarias respecto a España, y tasa de crecimiento de la renta por hogar en Canarias y España (\%, eje secundario)

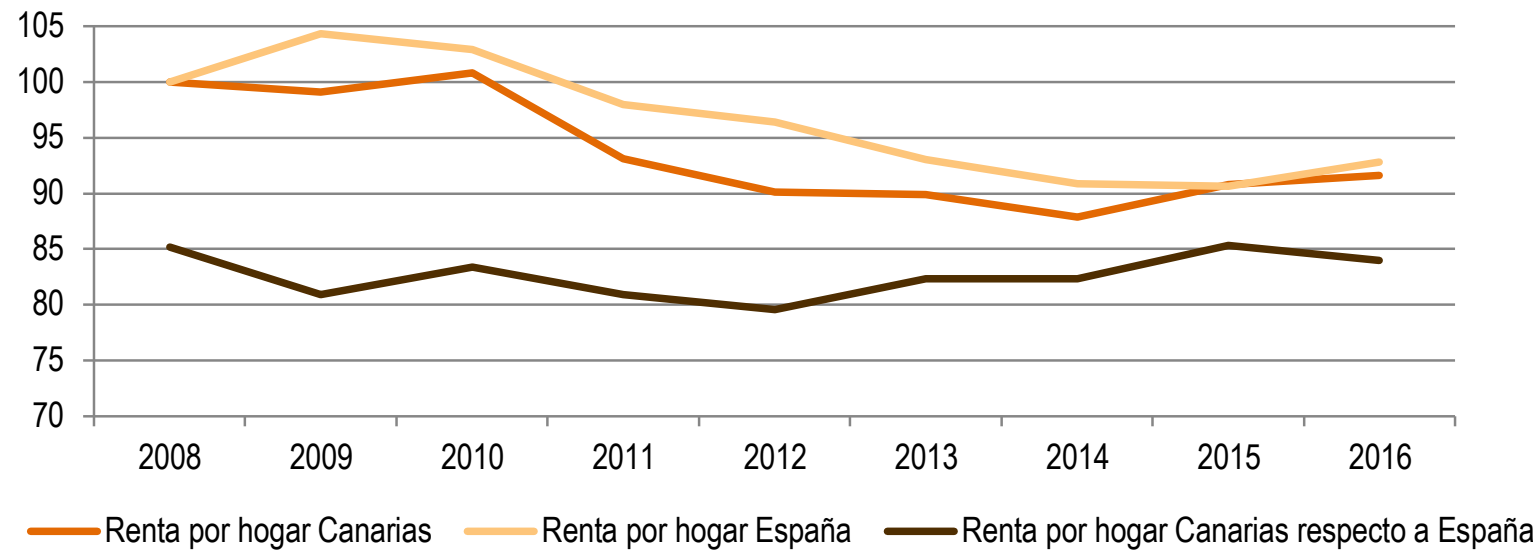

FUENTE: INE. Encuesta de Condiciones de Vida

NOTA: El cálculo de los indicadores de población ocupada y desempleada se ha realizado utilizando el promedio de los datos trimestrales ofrecidos por el INE

\subsection{Desigualdad de oportunidades y prosperidad económica}

Todos estos problemas que permanecieron ocultos tras el periodo de bonanza económica de mediados de los 90 hasta el año 2008 han sido puestos de relieve por la Gran Recesión. Canarias, al igual que gran parte del mundo desarrollado, es hoy más desigual de lo que era en 2008. Uno de los fenómenos asociados a esta desigualdad en auge, y que está más presente en las agendas de los países y las instituciones internacionales, es el de desigualdad de oportunidades (DO, de aquí en adelante). La incidencia de la DO en una sociedad se deriva de factores totalmente ajenos al control del individuo (sexo, lugar de nacimiento, barrio de residencia, colegio, nivel socioeconómico de los padres, etc.) que acaban determinando los resultados del individuo (estudios, ocupación, renta, etc.). El hecho de que la DO domine en la desigualdad total, por encima del talento, la capacidad o el esfuerzo del individuo (desigualdad de esfuerzos, DE, de aquí en adelante) deriva en sociedades alejadas de una estructura meritocrática, con un bajo grado de movilidad social intergeneracional (MOI, de aquí en adelante). Esto, de acuerdo con Marrero y Rodríguez (2012; 2013), no sólo es socialmente injusto, sino que además deriva en una asignación ineficiente del talento (favorece la acumulación de capital humano sólo por parte de aquellos individuos con orígenes sociales más acomodados), lo que acaba afectando al potencial de crecimiento económico de la propia economía.

El reto de los países para crear prosperidad económica dependerá, de acuerdo con Acemoglu y Robinson (2013), de su capacidad para crear un escenario que garantice la movilización del talento y las habilidades de un gran número de individuos, el que todos/as, independientemente de su origen social., puedan tener oportunidades de progreso, puedan participar con un mínimo de igualdad de oportunidades, puedan escoger su profesión y tengan incentivos a aprender, a formarse, a participar, a innovar, a emprender y a invertir.

\footnotetext{
2 Véase INE (2017) para una definición completa del concepto. También disponible en: goo.gl/f5jpDD
} 
La realidad social, sin embargo, tiende a parecerse más al primer escenario que al segundo ideal planteado sobre estas líneas. Ciertamente, la crisis no sólo ha puesto en relieve las disparidades antes ocultas -pero no por ello inexistentes- detrás de la bonanza económica, sino que además ha puesto en duda la capacidad de las instituciones para crear un entorno favorable a la igualdad de oportunidades. Cada vez son más los indicios en favor del atasco del 'ascensor social'; la probabilidad de ascenso social apenas ha cambiado; al tiempo que el reparto de la renta y su acumulación en un número menor de manos se ha hecho más evidente, las probabilidades de que los hijos/as de la clase baja y media opten a escalar socialmente es aún reducida en las islas. El origen social de las personas sigue teniendo un peso muy importante en España y en Canarias. Por ejemplo, la 'saturación' de titulados/as superiores, en un sistema que no genera el suficiente trabajo cualificado, que no se mueve lo suficientemente rápido hacia sectores y/o actividades de mayor valor añadido y conocimiento incorporado, se ha hecho evidente, 10 que en los últimos años ha desvirtuado en parte el 'premio de la educación'. Escenarios como este, caracterizados por la falta de inclusividad, suponen una amenaza tan importante como real de ruptura social, pérdida de legitimidad política y descrédito y desafección hacia las instituciones, así como la entrada en un peligroso círculo vicioso en el que mayor desigualdad conduce a un bucle de a mayor desigualdad de oportunidades y esta a su vez a mayor inmovilidad y menor crecimiento (Corak, 2013).

El objetivo del informe Desigualdad de oportunidades y movilidad intergeneracional en Canarias es el de subsanar la carencia -hasta ahora- de fuentes de información, así como aportar evidencias que ayuden a medir de forma rigurosa la DO y la $\mathrm{MOI}$ en Canarias. En este Informe, además de medir y analizar ambos indicadores para el archipiélago canario, se relacionan los resultados alcanzados por los individuos no sólo con sus circunstancias de partida, sino, además, con variables relacionadas con su fortaleza psicológica. Las 10 conclusiones y recomendaciones que se presentan a continuación constituyen los principales resultados obtenidos por el equipo investigador del Informe, así como 10 recomendaciones de política pensadas para combatir la DO y la inmovilidad social en Canarias. 


\section{La desigualdad de oportunidades en Canarias en 10 claves}

1. Desigualdad en la renta: Un reparto dispar con diferencias peligrosamente amplias en los extremos. La amenaza de casi un $45 \%$ de la población en riesgo de exclusión social

Canarias es una de las regiones españolas con mayores niveles de desigualdad. Los datos revelan que el $30 \%$ de la población con mayor nivel de ingresos capta el $50 \%$ de la renta total. Al comparar los extremos en la distribución de los ingresos, el 10\% de población con mayores ingresos tiene una renta media que es, aproximadamente, 7 veces la del $10 \%$ con menor nivel de ingresos (Gráfico 4). La desigualdad además, tal y como reflejan indicadores como la tasa de pobreza relativa o el porcentaje de población en riesgo de exclusión social (indicador AROPE) ha repuntado durante la última crisis económica, haciéndolo además por encima de la media nacional, lo que lleva a pensar que la situación de los habitantes de Canarias puede haberse vuelto más vulnerable a posibles variaciones externas futuras.

GRÁFICO 4: Distribución de la renta neta total de los individuos, según el decil de renta al que pertenecen

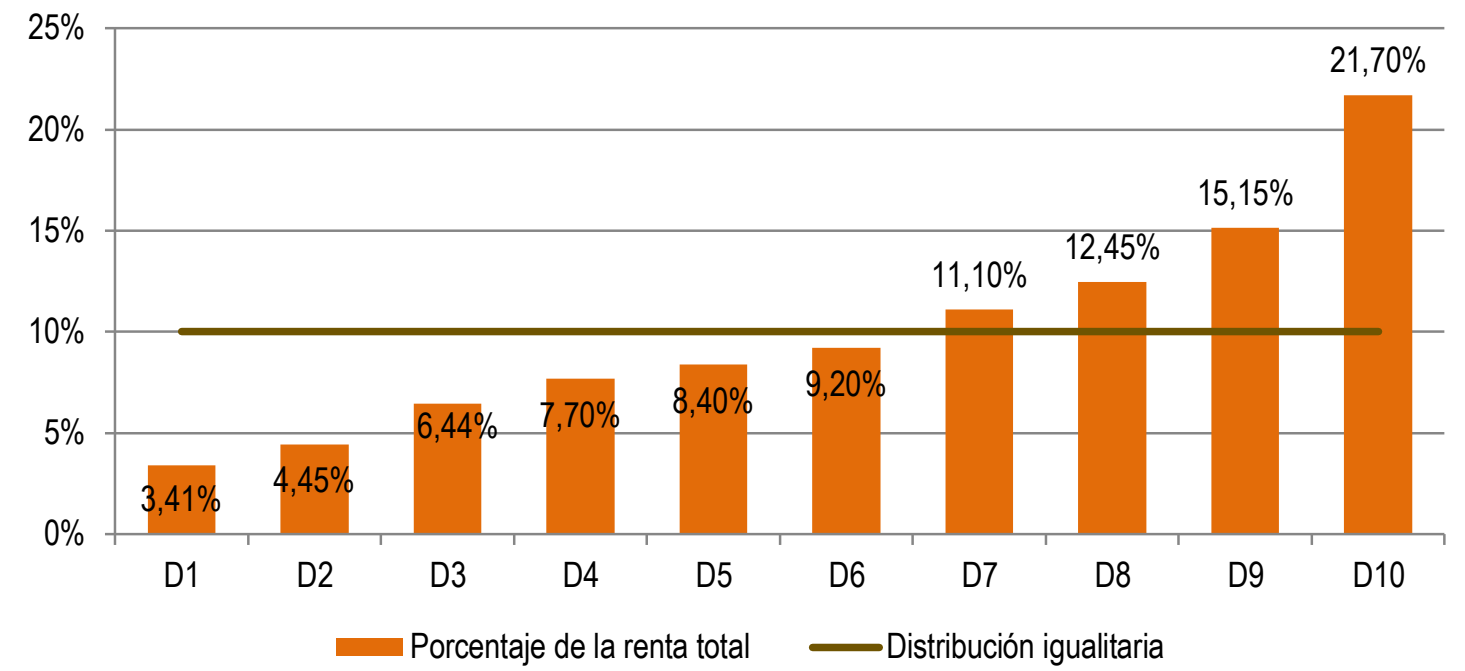

2. Diferencias en renta relevantes a partir del ciclo superior de educación secundaria. La ocupación y el efecto indirecto de la salud sobre los ingresos individuales

Los estudios alcanzados (años de escolarización equivalentes), el tipo de ocupación y el estado de salud del individuo son importantes para elevar los ingresos. Pero cabe matizar algunos aspectos. En primer lugar, los años de escolarización son relevantes solo a partir del ciclo superior de secundaria, muy especialmente, cuando el individuo alcanza los estudios terciarios (universitarios). En segundo lugar, una vez que tenemos en cuenta el tipo de ocupación, el estado de salud autopercibido del individuo deja de ser significativo, lo que reflejaría que la salud afecta a la renta, aunque tan solo a través del estatus ocupacional del individuo. En su conjunto, estas tres variables (ocupación, educación y estado de salud) explican en torno a un $20 \%$ del total de la variabilidad observada en la renta de los individuos. Esto implica que otros factores no observados tales como la calidad de la educación, las redes clientelares, u otros aspectos psicológicos de las personas, como su predisposición al esfuerzo, o su grado de fortaleza individual podrían tener una gran capacidad explicativa. 
3. La influencia de las circunstancias (1): Género, origen social, colegio y nivel socioeconómico de los padres como predictores de la renta del individuo

Son varias las circunstancias que explican de manera más significativa las diferencias en renta de los individuos considerados/as en la encuesta. La primera es el género; ser mujer supone en torno a un $15 \%$ menos de ingresos, y ello a pesar de que las mujeres poseen, en media (especialmente en las nuevas generaciones) un nivel educativo (en años de escolarización equivalentes) superior al de los hombres. La segunda de ellas, el origen de los individuos. Ser canario/a y haberse movido de la isla de origen, o ser originario/a de la Península son circunstancias que suponen casi un 20\%-25\% mayor de ingresos. En tercer lugar, proceder de una familia monoparental penaliza al individuo en un $15 \%$ de renta inferior a uno/a nacido/a en el seno de una familia nuclear tradicional. En cuarto lugar, la ocupación de los padres puede llegar a suponer un ingreso un $18 \%$ mayor si el padre del individuo contaba con una ocupación de estatus alto respecto a una de estatus bajo. En quinto lugar, haber tenido un mal estado de salud durante la infancia/adolescencia supone tener aproximadamente un $17 \%$ menos de ingresos. La sexta circunstancia, el haber ido a un colegio privado o concertado implica un $14 \%$ superior de ingresos respecto a los centros públicos. Finalmente, en séptimo y último lugar, un buen ambiente familiar durante la infancia (que los padres lean habitualmente, y/o inculquen a sus hijos/as la importancia del esfuerzo) también favorece un mayor nivel de ingresos, aunque su significatividad se pierde una vez que todas las circunstancias anteriores se incluyen.

\section{La influencia de las circunstancias (2): La desigualdad de oportunidades como amenaza al desarrollo en las Islas Canarias}

El conjunto de circunstancias consideradas explica el $14 \%$ de la variabilidad de la renta de los individuos en Canarias, un porcentaje algo inferior al alcanzado por el nivel de estudio y ocupación de los individuos. Esto implica que la DO estimada (una cota inferior de la DO) representa el $12 \%$ de la desigualdad total de Canarias (dependiendo del índice usado este porcentaje puede llegar a casi el 30\%). Este valor, aunque no directamente comparable con resultados existentes, está en línea con los estimados para España, aunque son muy superiores a los de las economías europeas con menor incidencia de la DO (por ejemplo, Dinamarca posee un ratio entorno al $5 \%$ ). Por islas, obtenemos que las islas pequeñas como el Hierro o la Gomera, la DO representa casi un 50\% sobre la desigualdad total. Estas islas, no obstante, tienen niveles de desigualdad similares (incluso inferiores, medida por el Coeficiente de Gini) a otras islas menores e incluso a las capitalinas. Con respecto a estas últimas, Tenerife registra un ratio de DO sobre la desigualdad total que ronda el $19 \%$, mientras que el de Gran Canaria es de aproximadamente el $11 \%$.

\section{Movilidad social intergeneracional en educación (1): Alta inmovilidad en los estratos superiores y mayor movilidad en los estratos bajos e intermedios}

Canarias presenta resultados de movilidad intergeneracional bajos, aunque algo superiores a los valores de Latinoamérica, por tomar una referencia distinta a España. Si comparamos los resultados de educación (padres versus hijo/a) obtenemos que, condicionado a que el padre tenga estudios primarios 0 secundarios, el porcentaje de individuos que alcanzan los estudios terciarios es aproximadamente del $21 \%$ y un $41 \%$, respectivamente; mientras que, si los padres tienen estudios terciarios, la probabilidad de que el hijo también alcance estudios superiores es cercana al $65 \%$. En el otro extremo, si los padres tienen estudios primarios, casi el $40 \%$ de los hijos/as acaban teniendo también estudios primarios. Estos porcentajes son similares si comparamos el estatus ocupacional del padre y del hijo/a. También existe evidencia de un "efecto de refuerzo" (Gráfico 5); el que ambos padres tengan estudios primarios (BB) supone que el hijo/a sólo tiene una probabilidad del $20 \%$ de alcanzar estudios terciarios y de más del $40 \%$ de quedarse con estudios primarios, mientras que estos porcentajes son del $26 \%$ y del $13 \%$ respectivamente si al menos uno de los dos progenitores tiene estudios secundarios. El mayor salto en este punto se observa cuando ambos padres tienen estudios terciarios; las probabilidades de que sus hijos/as alcancen los estudios terciarios es de entre el $60 \%$ si sólo uno de ellos alcanzó los estudios universitarios (A) y del $67 \%$ cuando ambos acudieron a la universidad (AA). 
GRÁFICO 5: Probabilidad condicionada de que el individuo alcance un determinado nivel de estudios, condicionada al nivel educativo de los padres (ambos) ${ }^{3}$

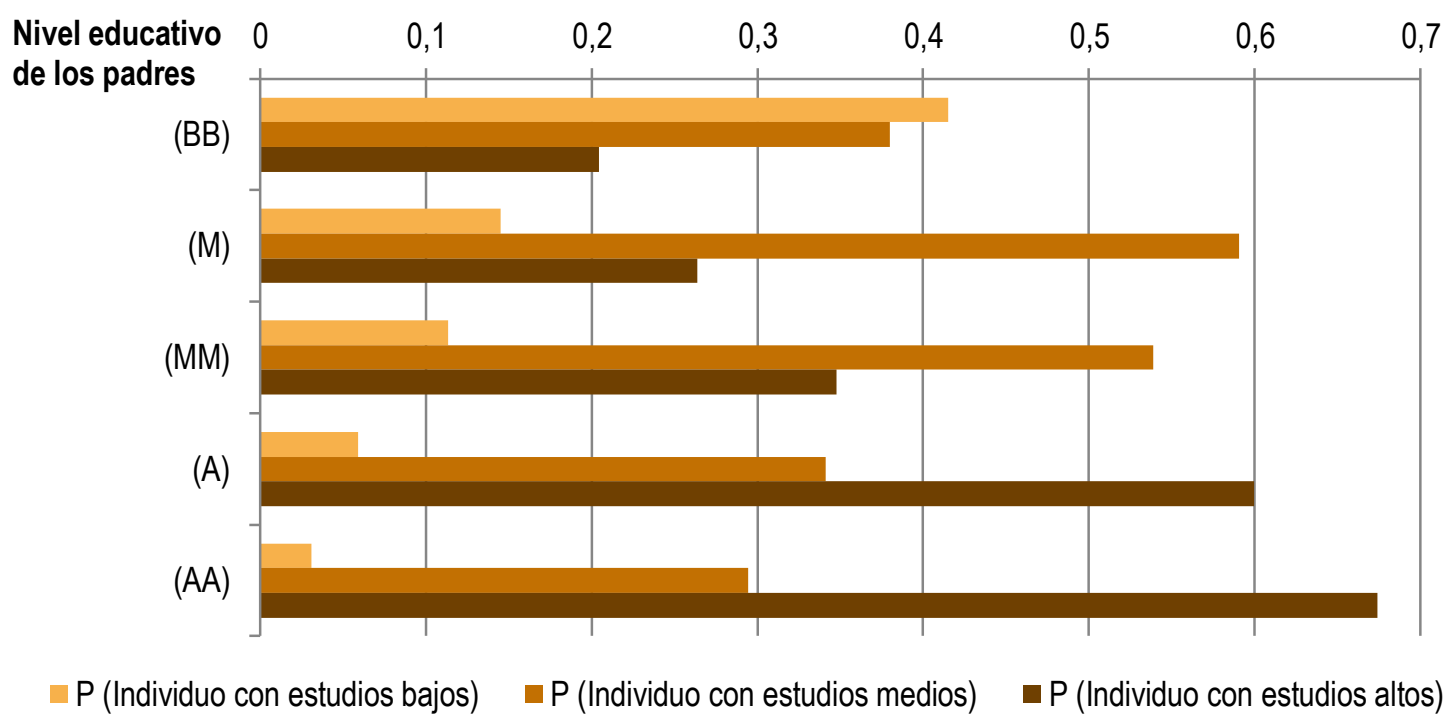

6. Movilidad social intergeneracional en educación (2): Mayor movilidad entre las nuevas generaciones, mayor inmovilismo en la transición hacia los estudios superiores

El grado de $\mathrm{MOl}$ en Canarias ha aumentado, especialmente en las nuevas generaciones respecto a las anteriores, un hecho también presente en muchos otros países. Por tanto, los logros de las nuevas generaciones dependen menos de los logros alcanzados previamente por sus padres. Esta evolución se debe, principalmente, a una mayor movilidad "hacia arriba", especialmente desde los estudios primarios a los secundarios y de los secundarios a los terciarios (gracias a una mayor universalidad de la educación superior). La mayor movilidad observada también se debe a un aumento de la movilidad "hacia abajo", desde los padres con estudios terciarios a hijos con estudios secundarios. La inmovilidad en la zona alta (alcanzar estudios terciarios cuando el padre tiene estudios terciarios) y en la zona baja, ha permanecido relativamente constante entre ambas generaciones.

\section{Perfil y fortaleza psicológica: a mejores condiciones socioeconómicas, mayor bienestar}

El perfil psicológico no es ajeno al contexto social en el que viven y se educan los individuos. Tomando como referencia los resultados sobre cómo los habitantes de Canarias se posicionan en torno a la felicidad, satisfacción, fortaleza y bienestar psicológico, encontramos que la renta del hogar es la más significativa a la hora de explicar su grado de bienestar psicológico. Así, a mejores condiciones económicas del hogar del encuestado, mejores estados psicológicos declaran estos. Por tanto, aquellas circunstancias que afecten más a la renta individual tendrán efectos indirectos más significativos sobre las variables de felicidad y bienestar psicológico. Es importante resaltar que estos efectos son de largo alcance. Así, por ejemplo, la capacidad de fortaleza personal se ve afectada por las circunstancias del individuo de manera directa, pero también de manera indirecta a través de la renta alcanzada, lo que a su vez, afecta a sus logros futuros (efecto multiplicador). Esta observación se vuelve particularmente relevante ante situaciones de crisis como las vividas recientemente.

\footnotetext{
3 La escala empleada para este gráfico considera a los individuos en función de si sus dos progenitores contaban ambos con estudios primarios (B), si el mayor nivel entre ambos era secundario $(M)$, si ambos alcanzaron los estudios secundarios (MM), si al menos uno de ellos estudió en la universidad (A), o si lo hicieron los dos (AA).
} 
8. Autopercepción de clase social: Predominio de la clase media. La mejora en la autopercepción económica actual (respecto a la juventud) va por grupos

La autopercepción de clase es un identificador condicionado por las trayectorias previas de los sujetos, así como por las percepciones subjetivas en torno a la propia estructura social. Los habitantes de Canarias se posicionan en esta dimensión de forma parecida al resto de la población española y europea. Se declaran mayormente de clase media. Sin embargo, y como tendencia encontramos que es en los grupos de estatus ocupacional medio y bajo donde más sienten los individuos que su situación no ha mejorado tanto como en el grupo de estatus ocupacional alto. Para el género femenino, hallamos casi exactamente la misma tendencia.

9. Fortaleza personal: Cuanto mejores son las condiciones socioeconómicas, más preparados nos vemos para afrontar situaciones y decisiones familiares

En general, la renta individual o del hogar, favorece estados de salud positivos, mayor felicidad personal, más satisfacción con su vida, y mejores estados psicológicos, con mayor fortaleza personal para acometer y tomar decisiones familiares y personales. Como estas situaciones están también muy condicionadas por el origen social familiar (circunstancias), nos enfrentamos a un problema de DO que repercute negativamente en la valoración y percepción que los ciudadanos tienen sobre la sociedad en la que viven.

10. Estado de salud: La mejor política de prevención, la adquisición de hábitos saludables desde edades tempranas

El estado de salud viene condicionado en gran medida por el nivel cultural y socioeconómico de la persona. En nuestro estudio concluimos que las circunstancias personales (nivel educativo y estatus ocupacional de los padres) influyen de forma significativa no sólo sobre la autopercepción del estado de salud actual de los individuos, sino que además afectan sus características físicas, como su estado de salud, hábitos alimenticios o peso. Al respecto, cabe decir que Canarias presenta una de las mayores tasas de sobrepeso y obesidad, tanto en población infantil como adulta, en España. La adquisición de hábitos saludables está altamente influenciada por el contexto familiar lo que nos lleva a pensar que la trasmisión de padres a hijos/as es un elemento clave para entender cómo combatir de forma eficaz -y temprana- la obesidad infantil. 
3. 10 recomendaciones de política para luchar contra la desigualdad de oportunidades en Canarias

\section{Luchar contra la desigualdad de oportunidades: Una cuestión de justicia social y eficiencia} económica

Una medida contra la desigualdad general puede afectar a diferentes tipos de desigualdad (por ejemplo, la DO o la desigualdad generada por diferentes niveles de talento o de esfuerzo, DE), las cuales a su vez, tal y como han demostrado otros trabajos previos, tienen impactos distintos sobre la economía (negativo la DO, y positiva la DE). Así, para combatir contra la DO, que es la considerada como más injusta y más ineficiente, se ha de combatir el origen del problema en su propia raíz.

\section{Actuar en el foco del problema: Corregir la desigualdad de oportunidades en origen}

Las políticas deberían encaminarse hacia los factores concretos que causan DO e inmovilidad social frente a políticas generales que afectan a toda la población con un resultado global incierto. Por ejemplo, a partir de actuaciones concretas sobre la igualdad de género, campañas de concienciación sobre la importancia de adquisición de unos buenos hábitos de salud, mejora del acceso a instalaciones deportivas, educativas y/o culturales, etc.

3. Luchar contra la corrupción o eliminar barreras a la creación de empresas: Medidas macroeconómicas para reducir la desigualdad de oportunidades

Gran parte del origen de la DO y de la inmovilidad social proviene de aspectos macroeconómicos (que no han sido tratados en este Informe) del país y de la región en la que se habita. Así, luchar contra la corrupción (vinculada en gran medida a las redes clientelares), eliminar obstáculos a la creación de empresas, reducir la capacidad de influencia de los grupos de interés, fomentar una mayor competencia en sectores concretos de la economía y finalmente, mejorar el acceso al mercado laboral de todos los colectivos poblacionales por igual, pueden ser algunas de las medidas requeridas para la reducción de la DO.

4. Mejorar la educación y las oportunidades en todos los grupos de edad: Uno de los canales clave para luchar contra la desigualdad de oportunidades

La educación es un aspecto clave como mecanismo para reducir la DO. De hecho, hemos visto que para el caso de Canarias la diferencia fundamental se da entre los estudios secundarios y los de FP superior y universitarios. Así, reducir la DO pasa inevitablemente por incentivar y promover el acceso a estas modalidades de formación a toda la población. Los sistemas de becas (condicionados a logros) y de créditos preferentes (con cierta carencia, de modo que el estudiante no comience a devolver hasta encontrar un empleo estable) y el aumento de plazas en los ciclos formativos de enseñanza profesional, previo estudio sobre la oferta formativa post-obligatoria por zonas, comarcas y municipios de las islas, pueden ser un buen sistema para fomentar la igualdad de oportunidades. Cuando los individuos no se hallen en edad escolar, los esfuerzos deberían centrarse en diseñar una oferta formativa de enseñanza profesional a través de la formación profesional ocupacional y de la formación profesional continua, sin descartar medidas que faciliten la educación de adultos.

5. Nivelando el escenario: El papel clave de la enseñanza pública en la igualdad de oportunidades

La mejora en la calidad de la enseñanza pública es fundamental para reducir el diferencial educativo entre centros (privados y públicos) y niveles (educación primaria y secundaria), en materia de recursos, planes formativos, ratio profesores/alumnos/as, absentismo de los docentes, etc., favoreciendo la igualdad de oportunidades. A este respecto, ciertos países referentes en la lucha contra la DO, como 
Finlandia, destinan más recursos a los centros educativos situados en aquellos barrios con mayor marginación y pobreza. Para la población no escolarizada (aquellos/as que tuvieron menores oportunidades, o incluso no dispusieron de oportunidades de acceso a la escolarización), habría que aplicar políticas de formación profesional ocupacional y/o continua, además de extender la oferta de espacios formativos en centros escolares e institutos con el apoyo y la participación de los ayuntamientos, al mismo tiempo que fomentar y aplicar medidas que refuercen la red pública de la enseñanza obligatoria, vigilando además las prácticas de selección de familias de la red privada.

\section{Conciliación laboral y reducción de la desigualdad de oportunidades: Igualdad de género, bajas por maternidad y paternidad, ayudas a familias monoparentales y por discapacidad}

Una circunstancia relevante es el género: según nuestras estimaciones, ser mujer supone, en media, entre un $14 \%$ y un $20 \%$ menos de ingresos que ser hombre. En muchos casos, esto puede deberse a decisiones respecto a la conciliación entre la vida personal y la profesional, en las cuales las mujeres se ven condicionadas a aceptar un contrato por un menor número de horas (a tiempo parcial), 0 a perder ciertos complementos salariales por productividad u horas extra. Nuestro Informe no es capaz de distinguir por fuentes el origen de la discriminación salarial. Sin embargo, si combinamos los resultados obtenidos en este Informe con los ofrecidos por otros estudios recientes, una fuente clara de DO está relacionada con los recursos destinados a la conciliación familiar y a una mayor protección de la mujer ante las bajas por embarazo y cuidado de menores o personas dependientes a su cargo. Aumentar el tiempo de baja maternal y dar facilidades (temporales) a los padres (tanto madre como padre) hasta los tres años de edad del hijo/a es una medida en pro de la igualdad de oportunidades. La ayuda por personas dependientes a cargo, también entraría en una categoría similar a la de igualdad de oportunidades. Respecto a las familias monoparentales, tal y como concluimos en este Informe, se trata de una circunstancia adversa respecto a los futuros ingresos de los individuos. Introducir medidas que permitan a los/as cabezas de familia de esta clase de unidades conciliar su vida familiar y la crianza y educación de sus hijos/as con el trabajo sería una medida adecuada para nivelar las desventajas con las que parten esta clase de individuos, así como sus descendientes.

\section{La importancia del ambiente familiar: Medidas de acción afirmativa y de fomento de la implicación familiar en la educación para mejorar el rendimiento escolar de los niños/as con peores circunstancias de partida}

El ambiente familiar en el que se cría un niño/a supone un factor relevante en sus posibilidades futuras de desarrollo. Conscientes de la dificultad de formular una propuesta de intervención en este ámbito, posiblemente, la mejor manera de hacerlo sea con acciones de educación familiar. En este sentido, cabe mencionar que algunas medidas de "acción afirmativa" implementadas en regiones desfavorecidas en países latinoamericanos han probado ser eficaces en la práctica. Así, por ejemplo, condicionar ciertas ayudas directas a los padres (para financiar libros, material escolar, transporte público o clases de refuerzo) a los logros y el rendimiento académico de los hijos/as (incluyendo criterios de asistencia, mejoría de notas, etc.) es una medida de "acción afirmativa" que ha demostrado ser de utilidad para reducir la DO en las zonas más desfavorecidas. Otras dos medidas relevantes podrían ser la subvención de plazas en las guarderías públicas y el ofrecer clases de refuerzo al alumnado que lo precise, desde los centros educativos y en horarios extraescolares. La primera medida, el favorecer un mayor acceso a la educación infantil (0-3 años) es un instrumento que claramente puede reducir el efecto de las circunstancias en las trayectorias individuales, mejorando el grado de igualdad de oportunidades, al mismo tiempo que se fomenta el acceso a la formación y al trabajo de las madres con menos recursos. La segunda consistiría en ofrecer un apoyo adicional a los estudiantes cuyas familias tengan dificultades para apoyar las tareas de sus hijos/as. Para ello será clave la colaboración de las autoridades locales, especialmente en las zonas más pobres y marginadas, a la hora de integrar más a los padres en la vida del centro y en la educación de sus hijos/as. 
8. Salud e igualdad de oportunidades: La importancia de la adquisición temprana de hábitos saludables

Impulsar intervenciones sanitarias y de salud pública que reduzcan las desigualdades en salud, así como vertebrar la salud como un eje trasversal, mediante un enfoque intersectorial de salud en todas las políticas. Favorecer la coordinación intersectorial para abordar los problemas de salud, reforzando las intervenciones a nivel local, unificando esfuerzos desde las distintas instituciones para garantizar la adquisición de hábitos saludables, incidiendo en una alimentación equilibrada y una actividad física regular como factores fundamentales en la lucha contra el sobrepeso y la obesidad.

9. Medir para corregir: Mejorar las estadísticas y asegurar fuentes de información estables es crucial para poder definir, implementar y evaluar políticas de igualdad de oportunidades

Los políticos necesitan información más precisa y detallada sobre la DO y la $\mathrm{MOI}$ en Canarias para poder diseñar planes de actuación específicos. En este sentido, se requieren estudios específicos que complementen las estadísticas realizadas por el ISTAC en Canarias, con soporte y apoyo de los ayuntamientos y los cabildos. También se necesitan grupos multidisciplinares de economistas, sociólogos, politólogos, psicólogos y de salud pública para poder entender y analizar las complejas relaciones existentes en problemáticas tan multidimensionales como la DO y la $\mathrm{MOI}$. Poder hacerlo de forma rigurosa y precisa implica la necesidad de creación y promoción de "laboratorios de análisis" sobre estos asuntos sociales, independientes 0 vinculados a grandes centros educativos como las universidades, tal y como ya existen en muchos otros países. En este sentido, en Canarias, la creación del Centro de Estudios de Desigualdad y Gobernanza de la Universidad de La Laguna (CEDESOG) constituye una primera y esperanzadora iniciativa en esta dirección.

10. Concienciar para luchar contra el problema: Ofrecer a la sociedad el conocimiento necesario para comprender la desigualdad de oportunidades y la movilidad intergeneracional

Se han de generar productos sencillos y programas de formación que tengan alcance social, para poder así trasmitir el conocimiento a la población en general y a la clase política en particular, sobre la distinción entre los distintos tipos de desigualdad, los determinantes de la DO, la relevancia de mejorar el grado de $\mathrm{MOI}$ en la sociedad, y el analizar, evaluar y realizar un riguroso seguimiento de las políticas a aplicar. 


\section{Bibliografía}

Acemoglu, D., \& Robinson, J. A. (2013). Por qué fracasan los países. Los orígenes del poder, la prosperidad y la pobreza. Madrid: Deusto.

Agencia EFE. (2017). La temporalidad en el empleo es del 32,5\% en Canarias. Obtenido de www.canarias7.es/economia/empleo/: goo.gl/dC1Tnz

Corak, M. (2013). Income Inequality, Equality of Opportunity, and Intergenerational Mobility. Journal of Economic Perspectives, 27, 79-102.

Domínguez, Í. (2016). El ascensor social se atasca. Obtenido de politica.elpais.com: goo.gl//ZZCd1t

INE. (2016). Indicadores de condiciones de vida. Condiciones materiales de vida. Condiciones económicas. Renta disponible ajustada de los hogares. Obtenido de ine.es/ss/Satellite?L: goo.gl/zT6PnB

INE. (2017). Encuesta de población activa. Resultados por comunidades autónomas. Obtenido de ine.es/dyngs//NEbase/es/operacion.htm: goo.g//Ugbe6H

INE. (2017). Resultados por comunidades autónomas. Renta anual media (año anterior a la entrevista). Renta por hogar por comunidades autónomas. Obtenido de ine.es/jaxiT3/Datos.htm?t=9949: Igoo.gl/SA6sVU

INE. (2017). Riesgo de pobreza y/o exclusión social (estrategia Europa 2020). Indicador AROPE. Obtenido de ine.es/ss/Satellite?L: goo.gl/f5jpDD

INE. (2017). Encuesta anual de estructura salarial. Serie 2008-2015. Resultados por CC.AA. Ganancia media anual por trabajador. Obtenido de ine.es/jaxi/Datos.html: goo.gl/TZadep

ISTAC. (2017). PIB. Indicadores según comunidades autónomas y años (Base 2010). Obtenido de gobiernodecanarias.org/istac/jaxi-istac/tabla.do: goo.gl/MUy6pG

Marrero Díaz, G. A., Betancort Montesinos, M., Cabrera Rodríguez, L. J., Darias Curvo, S., Pérez Sánchez, C. N., Rodríguez Hernández, J. G., y otros. (2017). Desigualdad de oportunidades y movilidad intergeneracional en Canarias. Santa Cruz de Tenerife: Fundación Cajacanarias.

Marrero, G. A., \& Rodríguez, J. G. (2012). Inequality of opportunity in Europe. Review of Income and Wealth(58), 597-621.

Padrón Marrero, D., Martínez García, J. S., Gutiérrez Hernández, P., Godenau, D., Hernández Guerra, A. M., Rocío Salvo, C., y otros. (2016). Desigualdad, pobreza y cohesión social en Canarias. Análisis de su incidencia y distribución entre la población canaria. Gobierno de Canarias, Comisionado de Inclusión Social y Lucha contra la Pobreza, Santa Cruz de Tenerife. 



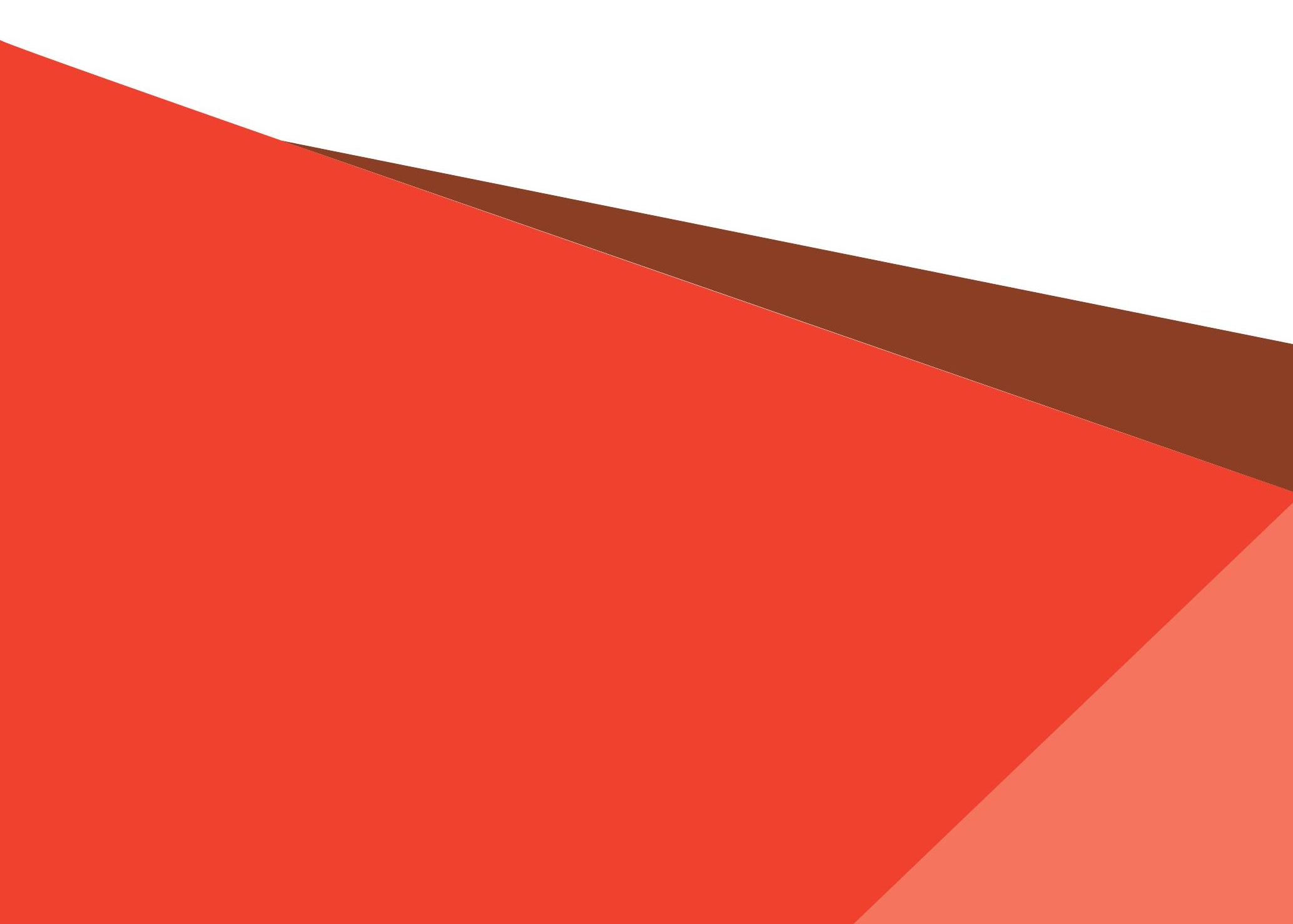

